Journal of Organometallic Chemistry, 263 (1984) 183-192

Elsevier Sequoia S.A., Lausanne - Printed in The Netherlands

\title{
UNTERSUCHUNG DER EIGENSCHAFTEN EINES UNGEWÖHNLICHEN DISCHWEFELLIGANDEN: VERGLEICHENDE BETRACHTUNG DER CHEMIE VON $\left(\mathrm{C}_{5} \mathrm{Me}_{5}\right)_{2} \mathrm{Cr}_{2}\left\{\left(\mu, \eta^{1}-\mathrm{S}_{2}\right)\left(\mu, \eta^{2}-\mathrm{S}_{2}\right)(\mu-\mathrm{S})\right\}$ und $\left(\mathrm{C}_{5} \mathrm{Me}_{5}\right)_{2} \mathrm{Mo}_{2}\left(\mu, \eta^{2}-\mathrm{S}_{2}\right)(\mu-\mathrm{S})_{2}$
}

HENRI BRUNNER, HEIKE KAUERMANN, WALTER MEIER Und JOACHIM WACHTER ${ }^{\star}$ Institut für Anorganische Chemie der Universität Regensburg, Universitätsstr. 31, D.8400 Regensburg (B.R.D.)

(Eingegangen den 28. September 1983)

\section{Summary}

The uncoordinated sulphur of the iso- $\mu\left(\eta^{\prime}-\mathrm{S}_{2}\right)$ ligand in $\left(\mathrm{C}_{5} \mathrm{Me}_{5}\right)_{2} \mathrm{Cr}_{2} \mathrm{~S}_{5}$ is easily abstracted by $\mathrm{PPh}_{3}$ causing formation of $\left(\mathrm{C}_{5} \mathrm{Me}_{5}\right)_{2} \mathrm{Cr}_{2} \mathrm{~S}_{4}$, isoelectronic with $\left(\mathrm{C}_{5} \mathrm{Me}_{5}\right)_{2} \mathrm{Mo}_{2}\left(\mu-\mathrm{S}_{2}\right)(\mu-\mathrm{S})_{2}$. From the nature of the products $\left[\left(\mathrm{C}_{5} \mathrm{Me}_{5}\right)_{2} \mathrm{Cr}_{2} \mathrm{~S}_{5} \mathrm{H}\right] \mathrm{PF}_{6}$, $\left[\left(\mathrm{C}_{5} \mathrm{Me}_{5}\right)_{2} \mathrm{Cr}_{2} \mathrm{~S}_{5} \mathrm{CH}_{3}\right] \mathrm{PF}_{6},\left[\left(\mathrm{C}_{5} \mathrm{Me}_{5}\right)_{2} \mathrm{Cr}_{2} \mathrm{~S}_{4} \mathrm{CH}_{3}\right] \mathrm{PF}_{6}$ and $\left[\left(\mathrm{C}_{5} \mathrm{Me}_{5}\right)_{2} \mathrm{Mo}_{2} \mathrm{~S}_{4} \mathrm{CH}_{3}\right] \mathrm{PF}_{6}$ formed from these complexes by reaction with $\mathrm{CF}_{3} \mathrm{CO}_{2} \mathrm{H}$ and $\mathrm{CH}_{3} \mathrm{I}$ a decrease in nucleophilicity is deduced for $\mu\left(\eta^{1}-\mathrm{S}_{2}\right)>\mu-\mathrm{S}>\mu\left(\eta^{2}-\mathrm{S}_{2}\right)$. The reaction of $\left(\mathrm{C}_{5} \mathrm{Me}_{5}\right)_{2} \mathrm{Mo}_{2} \mathrm{~S}_{4}$ with $\mathrm{C}_{5} \mathrm{H}_{5}(\mathrm{CO})_{2} \mathrm{Mn}(\mathrm{THF})$ gives a complex of composition $\left(\mathrm{C}_{5} \mathrm{Me}_{5}\right)_{2} \mathrm{Mo}_{2}\left(\mathrm{C}_{5} \mathrm{H}_{5}\right) \mathrm{Mn}(\mathrm{CO})_{2} \mathrm{~S}_{4}$, whereas $\left(\mathrm{C}_{5} \mathrm{Me}_{5}\right)_{2} \mathrm{Cr}_{2} \mathrm{~S}_{5}$ gives only the known complexes $\left[\mathrm{C}_{5} \mathrm{H}_{5}(\mathrm{CO})_{2} \mathrm{Mn}\right]_{2} \mathrm{~S}_{2}$ and $\left[\mathrm{C}_{5} \mathrm{H}_{5}(\mathrm{CO})_{2} \mathrm{Mn}\right]_{2} \mathrm{~S}$ together with $\left(\mathrm{C}_{5} \mathrm{Me}_{5}\right)_{2} \mathrm{Cr}_{2} \mathrm{~S}_{4}$. In the reaction with $\mathrm{H}_{2}\left(150 \mathrm{bar} / 80^{\circ} \mathrm{C}\right)\left(\mathrm{C}_{5} \mathrm{Me}_{5}\right)_{2} \mathrm{Mo}_{2} \mathrm{~S}_{4}$ forms $\left(\mathrm{C}_{5} \mathrm{Me}_{5}\right)_{2} \mathrm{Mo}_{2} \mathrm{~S}_{4} \mathrm{H}_{2}$, in contrast to $\left(\mathrm{C}_{5} \mathrm{Me}_{5}\right)_{2} \mathrm{Cr}_{2} \mathrm{~S}_{4}$ and $\left(\mathrm{C}_{5} \mathrm{Me}_{5}\right)_{2} \mathrm{Cr}_{2} \mathrm{~S}_{5}$ which give the cubane type cluster $\left(\mathrm{C}_{5} \mathrm{Me}_{5}\right)_{4} \mathrm{Cr}_{4} \mathrm{~S}_{4}$.

\section{Zusammenfassung}

Der nicht koordinierte Schwefel des iso- $\mu\left(\eta^{1}-\mathrm{S}_{2}\right)$-Liganden in $\left(\mathrm{C}_{5} \mathrm{Me}_{5}\right)_{2} \mathrm{Cr}_{2} \mathrm{~S}_{5}$ lässt sich leicht mit $\mathrm{PPh}_{3}$ unter Bildung des zu $\left(\mathrm{C}_{5} \mathrm{Me}_{5}\right)_{2} \mathrm{Mo}_{2}\left(\mu-\mathrm{S}_{2}\right)(\mu-\mathrm{S})_{2}$ isoelektronischen Komplexes $\left(\mathrm{C}_{5} \mathrm{Me}_{5}\right)_{2} \mathrm{Cr}_{2} \mathrm{~S}_{4}$ abstrahieren. Aus der Natur der aus diesen Komplexen durch Umsetzung mit $\mathrm{CF}_{3} \mathrm{CO}_{2} \mathrm{H}$ und $\mathrm{CH}_{3} \mathrm{I}$ gebildeten Produkte $\left[\left(\mathrm{C}_{5} \mathrm{Me}_{5}\right)_{2} \mathrm{Cr}_{2} \mathrm{~S}_{5} \mathrm{H}\right] \mathrm{PF}_{6}, \quad\left[\left(\mathrm{C}_{5} \mathrm{Me}_{5}\right)_{2} \mathrm{Cr}_{2} \mathrm{~S}_{5} \mathrm{CH}_{3}\right] \mathrm{PF}_{6}, \quad\left[\left(\mathrm{C}_{5} \mathrm{Me}_{5}\right)_{2} \mathrm{Cr}_{2} \mathrm{~S}_{4} \mathrm{CH}_{3}\right] \mathrm{PF}_{6}$ und $\left[\left(\mathrm{C}_{5} \mathrm{Me}_{5}\right)_{2} \mathrm{Mo}_{2} \mathrm{~S}_{4} \mathrm{CH}_{3}\right] \mathrm{PF}_{6}$ lässt sich eine Abnahme der Nukleophilie für $\mu\left(\eta^{1}-\mathrm{S}_{2}\right)$ $>\mu-\mathrm{S}>\mu\left(\eta^{2}-\mathrm{S}_{2}\right)$ folgern. Die Reaktion von $\left(\mathrm{C}_{5} \mathrm{Me}_{5}\right)_{2} \mathrm{Mo}_{2} \mathrm{~S}_{4}$ mit $\mathrm{C}_{5} \mathrm{H}_{5}(\mathrm{CO})_{2^{-}}$ $\mathrm{Mn}(\mathrm{THF})$ ergibt einen Komplex der Zusammensetzung $\left(\mathrm{C}_{5} \mathrm{Me}_{5}\right)_{2} \mathrm{Mo}_{2^{-}}$ $\left(\mathrm{C}_{5} \mathrm{H}_{5}\right) \mathrm{Mn}(\mathrm{CO})_{2} \mathrm{~S}_{4}$, während $\left(\mathrm{C}_{5} \mathrm{Me}_{5}\right)_{2} \mathrm{Cr}_{2} \mathrm{~S}_{5}$ nur die bereits bekannten Komplexe $\left[\mathrm{C}_{5} \mathrm{H}_{5}(\mathrm{CO})_{2} \mathrm{Mn}\right]_{2} \mathrm{~S}_{2}$ und $\left[\mathrm{C}_{5} \mathrm{H}_{5}(\mathrm{CO})_{2} \mathrm{Mn}\right]_{2} \mathrm{~S}$ liefert, zusammen mit $\left(\mathrm{C}_{5} \mathrm{Me}_{5}\right)_{2} \mathrm{Cr}_{2} \mathrm{~S}_{4}$. Die Hochdruckreaktion von $\left(\mathrm{C}_{5} \mathrm{Mc}_{5}\right)_{2} \mathrm{Mo}_{2} \mathrm{~S}_{4}$ mit $\mathrm{H}_{2}\left(150 \mathrm{bar} / 80^{\circ} \mathrm{C}\right)$ erfolgt unter $\mathrm{H}_{2}$-Aufnahme zu $\left(\mathrm{C}_{5} \mathrm{Me}_{5}\right)_{2} \mathrm{Mo}_{2} \mathrm{~S}_{4} \mathrm{H}_{2} ;\left(\mathrm{C}_{5} \mathrm{Me}_{5}\right)_{2} \mathrm{Cr}_{2} \mathrm{~S}_{4}$ und $\left(\mathrm{C}_{5} \mathrm{Me}_{5}\right)_{2} \mathrm{Cr}_{2} \mathrm{~S}_{5}$ liefern dagegen den Cubancluster $\left(\mathrm{C}_{5} \mathrm{Me}_{5}\right)_{4} \mathrm{Cr}_{4} \mathrm{~S}_{4}$. 
Die Reaktion von Cyclooctaschwefel, $\mathrm{S}_{8}$, mit den zweikernigen Übergangsmetallcarbonylderivaten $\left[\eta^{5}-\mathrm{C}_{5} \mathrm{Me}_{5} \mathrm{M}(\mathrm{CO})_{2}\right]_{2}(\mathrm{M}=\mathrm{Cr}$, Mo, W) zeichnet sich durch eine Fülle von Produkten aus, die durch unterschiedliche Kombination verschiedener Ligandenfunktionen sowohl des einatomigen als auch des zweiatomigen Schwefels charakterisiert sind [1,2]. Besonderes Augenmerk verdient dabei der aus $\left[\mathrm{C}_{5} \mathrm{Me}_{5} \mathrm{Cr}(\mathrm{CO})_{2}\right]_{2}$ hervorgegangene Komplex $\left(\mathrm{C}_{5} \mathrm{Me}_{5}\right)_{2} \mathrm{Cr}_{2} \mathrm{~S}_{5}$, der neben einem $\mu$-Sund einem $\mu\left(\eta^{2}-S_{2}\right)$-Liganden das bisher einzigartige Strukturelement einer iso- $\mu\left(\eta^{1}-\right.$ $S_{2}$ )-Brücke beinhaltet [1]. Diese Anordnung ist umso überraschender, als eine Kombination aus zwei $\mu$-S- und einem $\mu\left(\eta^{2}-S_{2}\right)$-Liganden, wie sie in $\left(\mathrm{C}_{5} \mathrm{Me}_{5}\right)_{2} \mathrm{Mo}_{2}\left(\mu-\mathrm{S}_{2}\right)(\mu-\mathrm{S})_{2}$ anzutreffen ist [2], dem Zentralmetall genauso zu einer Edelgaskonfiguration verhelfen würde. Beide Komplextypen sind als schwefelreiche Substrate zum Aufbau von cubanartigen Clustern mit einem $\mathrm{M}_{4} \mathrm{~S}_{4}$-Kern geeignet [3]. In diesen Versuchen machte sich bereits die leichte Abspaltbarkeit des "freien", nicht-koordinierten Schwefels des $\mu\left(\eta^{1}-\mathrm{S}_{2}\right)$-Liganden in $\left(\mathrm{C}_{5} \mathrm{Me}_{5}\right)_{2} \mathrm{Cr}_{2} \mathrm{~S}_{5}$ bemerkbar. Weitere Untersuchungen sollen nun gezielt die Reaktivität dieser Gruppierung erhellen, wobei als Vergleichsmaterial der strukturell nahe verwandte Komplex $\left(\mathrm{C}_{5} \mathrm{Me}_{5}\right)_{2} \mathrm{Mo}_{2}\left(\mu-\mathrm{S}_{2}\right)(\mu-\mathrm{S})_{2}$ herangezogen wird sowie dessen in dieser Arbeit erstmals beschriebener homologer Chromkomplex I.

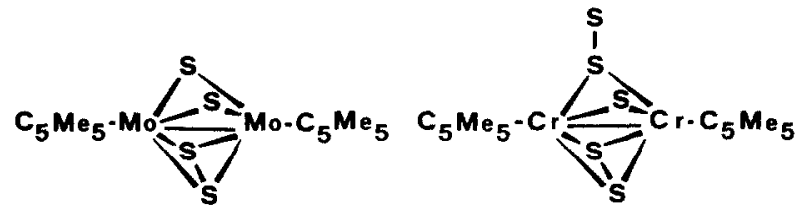

\section{Präparative Ergebnisse}

Reaktion von $\left(\mathrm{C}_{5} \mathrm{Me}_{5}\right)_{2} \mathrm{Cr}_{2} \mathrm{~S}_{5}$ mit $\mathrm{PPh}_{3}$

$\left(\mathrm{C}_{5} \mathrm{Me}_{5}\right)_{2} \mathrm{Cr}_{2} \mathrm{~S}_{5}$ reagiert mit einem Moläquivalent $\mathrm{PPh}_{3}$ in Toluol bei $45^{\circ} \mathrm{C}$ unter Schwefelabstraktion zum blaugrünen Komplex $\left(\mathrm{C}_{5} \mathrm{Me}_{5}\right)_{2} \mathrm{Cr}_{2} \mathrm{~S}_{4}$. Das im Vergleich zur Ausgangsverbindung wesentlich vereinfachte IR-Spektrum im Bereich der Metall-Schwefelbrücken-Schwingungen legt die Eliminierung des freien, nicht koordinierten Schwefels des iso- $\mu, \eta^{1}$-Liganden nahe, $d$. h. es würde eine zu $\left(\mathrm{C}_{5} \mathrm{Me}_{5}\right)_{2} \mathrm{Mo}_{2}\left(\mu-\mathrm{S}_{2}\right)(\mu-\mathrm{S})_{2}$ vergleichbare Ligandenanordnung erreicht. Eine ähnliche Reduzierung des Schwefelgehaltes von (i-PropC $\left.\mathrm{C}_{5} \mathrm{H}_{4}\right)_{2} \mathrm{~V}_{2} \mathrm{~S}_{5}$ (dieser Komplex enthält neben einer $\mu$-S- und einer $\mu, \eta^{2}-S_{2}$-Gruppe noch eine syn- $\eta^{1}-S_{2}$-Brücke [4]) mit Hilfe von $\mathrm{PBu}_{3}$ führt zu (i-Prop $\left.\mathrm{C}_{5} \mathrm{H}_{4}\right)_{2} \mathrm{~V}_{2} \mathrm{~S}_{4}$. Diese Reaktion verläuft unter Abbau der $\eta^{2}-S_{2}$-Brücke, während der $\eta^{1}-S_{2}$-Ligand erhalten bleibt [5]. In der resultierenden Ligandenanordnung leisten alle Schwefelatome einen gleichmässigen Stabilisierungsbeitrag über $\pi$-Wechselwirkungen, wodurch ein auf reinen $V_{-}$-S- $\sigma$-Bindungen basierendes starkes Elektronendefizit umgangen wird. Im Gegensatz zu diesem Befund lassen sich die Strukturen von $\left(\mathrm{C}_{5} \mathrm{Me}_{5}\right)_{2} \mathrm{Cr}_{2} \mathrm{~S}_{4}$ und $\left(\mathrm{C}_{5} \mathrm{Me}_{5}\right)_{2} \mathrm{Mo}_{2} \mathrm{~S}_{4}$ weitgehend durch Metall-Schwefel- $\sigma$-Donor-Wechselwirkungen beschreiben.

\section{Versuche zur Protonierung mit Trifluoressigsäure}

Aus einer Toluollösung von $\left(\mathrm{C}_{5} \mathrm{Me}_{5}\right)_{2} \mathrm{Cr}_{2} \mathrm{~S}_{5}$ erhält man mit einem Überschuss an $\mathrm{CF}_{3} \mathrm{CO}_{2} \mathrm{H}$ ein grünes $\mathrm{O}$, das sich durch $\mathrm{Umfällen}$ aus ethanolischer $\mathrm{NH}_{4} \mathrm{PF}_{6}$-Lösung 
TABELLE 1

IR-SPEKTREN DER KOMPLEXE I-VIII $\left(\mathrm{KBr}, \mathrm{cm}^{-1}\right)^{a}$

\begin{tabular}{lcrllll}
\hline & $\nu(\mathrm{CO})$ & $\nu(\mathrm{SH})$ & $\nu(\mathrm{CH})$ & $\nu(\mathrm{PF})$ & $\nu(\mathrm{M}-\mathrm{S}-\mathrm{M})$ & sonstige \\
\hline I & - & - & $1374,1023 \mathrm{~s}$ & - & $447 \mathrm{~m}, 400 \mathrm{vw}$ & $512 \mathrm{vw}$ \\
II & - & - & $1383 \mathrm{~m}, 1022 \mathrm{w}$ & $841 \mathrm{vs}, 554 \mathrm{~m}$ & - & - \\
III & - & - & $1381 \mathrm{~m}, 1028 \mathrm{w}$ & $843 \mathrm{~s}, 556 \mathrm{~m}$ & - & - \\
IV & - & - & $1382 \mathrm{~m}, 1019 \mathrm{w}$ & $839 \mathrm{vs}, 553 \mathrm{~m}$ & $463 \mathrm{vw}$ & $418 \mathrm{vw}$ \\
V & - & - & $1386 \mathrm{~m}, 1023 \mathrm{w}$ & $840 \mathrm{vs}, 558 \mathrm{~m}$ & $447 \mathrm{vw}$ & - \\
VI & $1945,1894 \mathrm{vs}, 1864 \mathrm{~m}$ & - & $1373 \mathrm{~s}, 1015 \mathrm{~m}$, & - & - & $628 \mathrm{~m}, 582 \mathrm{~s}, 552 \mathrm{~m}$, \\
& $1945 \mathrm{vs}, 1900 \mathrm{~s}{ }^{b}$ & & $816 \mathrm{~s}$ & & & $492 \mathrm{~m}, 475 \mathrm{~m}, 434 \mathrm{~m}$, \\
& & & & & & $355 \mathrm{w}$ \\
VII & - & - & $1376 \mathrm{~s}, 1022 \mathrm{~m}$ & - & $399 \mathrm{w}$ & $322 \mathrm{w}$ \\
VIII & - & $2419 \mathrm{w}$ & $1378 \mathrm{~s}, 1023 \mathrm{~m}$ & - & $417 \mathrm{~m}$ & $380 \mathrm{vw}$ \\
\hline
\end{tabular}

a Beckman 4240 Spektrophotometer. ${ }^{b}$ In Toluol.

als das grüne Komplexsalz II isolieren lässt. Während sich das $\mathrm{PF}_{6}$-Anion der resultierenden Verbindung II im IR-Spektrum (Tab. 1) durch seine P-F-Schwingungen verrät, lässt sich aus dem FD-Massenspektrum des Kations entnehmen, dass das ursprüngliche $\mathrm{S}_{5}$-Komplexgerüst bei der Protonierung erhalten geblieben ist. Allerdings macht sich bei erhöhter Fadenheizung eine Tendenz zur Schwefelabspaltung bemerkbar. Auf die Anzahl eingeführter Protonen kann nur indirekt geschlossen werden, da diese weder aus dem IR- noch aus dem ${ }^{1}$ H-NMR-Spektrum ersichtlich sind. Trotzdem kann indirekt auf eine Einfachprotonierung geschlossen werden, da die Umsetzung von $\left(\mathrm{C}_{5} \mathrm{Me}_{5}\right)_{2} \mathrm{Mo}_{2} \mathrm{~S}_{4}$ mit $\mathrm{CF}_{3} \mathrm{CO}_{2} \mathrm{H}$ unter den gleichen Bedingungen keinen isolierbaren Komplex liefert. In diesem Fall lässt sich nur eine reversible Farbänderung von blau nach violett beobachten, was mit einer nicht ausreichenden Basizität der $\mu$-S- bzw. $\mu\left(\eta^{2}-S_{2}\right)$-Liganden zu vereinbaren ist.

\section{Methylierungsreaktionen mit Methyliodid}

Die Methylierung von $\left(\mathrm{C}_{5} \mathrm{Me}_{5}\right)_{2} \mathrm{Mo}_{2} \mathrm{~S}_{4}$ mit einem Überschuss an Methyliodid führt über ein dunkelgrünes Pulver nach Umfällen aus ethanolischer $\mathrm{NH}_{4} \mathrm{PF}_{6}$-Lösung zu dem dunkelbraunen Salz $\left[\left(\mathrm{C}_{5} \mathrm{Me}_{5}\right)_{2} \mathrm{Mo}_{2} \mathrm{~S}_{4} \mathrm{Me}\right]^{+} \mathrm{PF}_{6}{ }^{-}$(III). Die Einführung nur einer Methylgruppe wird durch das ${ }^{1} \mathrm{H}$-NMR-Spektrum bewiesen, in dem die Methylgruppen des $\mathrm{C}_{5} \mathrm{Me}_{5}$-Restes mit der der $\mathrm{SCH}_{3}-\mathrm{Gruppe}$ ein 10/1-Verhältnis

TABELLE 2

${ }^{1}$ H-NMR-PARAMETER ( $\delta$-Werte in ppm, i-TMS, alle Signale Singuletts)

\begin{tabular}{|c|c|c|c|c|c|}
\hline & SH & $\mathrm{SCH}_{3}$ & $\mathrm{CH}_{3}$ & $\mathrm{C}_{5} \mathrm{H}_{5}$ & Lösungsmittel/Gerät \\
\hline$\overline{\mathrm{I}}$ & & & 1.98 & & $\mathrm{CDCl}_{3} /$ Varian T-60 \\
\hline II & $a$ & & 2.27 & & Aceton- $d_{6} /$ Bruker WH 90 \\
\hline III & & 1.33 & 2.33 & & $\mathrm{CDCl}_{3} /$ Varian T-60 \\
\hline IV & & 1.51 & 2.16 & & $\mathrm{CDCl}_{3} /$ Varian $\mathrm{T}-60$ \\
\hline \multirow[t]{2}{*}{ V } & & 2.44 & 2.28 & & Aceton- $d_{6} /$ Bruker WH 90 \\
\hline & & 1.65 & 2.22 & & \\
\hline VI & & & 1.96 & 4.29 & $\mathrm{CDCl}_{3} /$ Varian T-60 \\
\hline VIII & $-2.02,-1.95$ & & 2.22 & & $\mathrm{CDCl}_{3} /$ Varian T-60 \\
\hline
\end{tabular}

a Nicht auffindbar. 
bilden. Gegenüber neutralen thiolatoverbrückten Komplexen, wie z.B. $\left(\mathrm{C}_{5} \mathrm{H}_{5}\right)_{2} \mathrm{Mo}_{2}-$ $(\mu-\mathrm{S})_{2}(\mu-\mathrm{SMe})_{2}[6]$ ist eine Tieffeldverschiebung der $\mathrm{SCH}_{3}$-Protonen von ca. $2 \mathrm{ppm}$ $\mathrm{zu}$ beobachten, d.h. die positive Ladung am Schwefel bewirkt eine deutliche Entschirmung. Aus der Tatsache, dass sich $\left(\mathrm{C}_{5} \mathrm{Me}_{5}\right)_{2} \mathrm{Mo}_{2}\left(\mu-\mathrm{S}_{2}\right) \mathrm{S}_{2}$ [2] unter den gleichen Bedingungen wie oben quantitativ zurückgewinnen lässt, schliessen wir, dass die $\eta^{2}$-Disulfidbrücke ebenso wie terminale $\mathrm{Mo}=\mathrm{S}$-Liganden gegenüber $\mathrm{CH}_{3} \mathrm{I}$ inert ist. Eventuelle Inversionsisomere am neugeschaffenen $\mu$-SCH${ }_{3}$-Liganden sind nicht zu beobachten, da im ${ }^{1} \mathrm{H}-\mathrm{NMR}$-Spektrum die Singuletts für beide Methylgruppen bis $-77^{\circ} \mathrm{C}\left(\mathrm{CD}_{2} \mathrm{Cl}_{2}\right)$ unverändert erhalten bleiben.

Analog verläuft die Methylierung von $\left(\mathrm{C}_{5} \mathrm{Me}_{5}\right)_{2} \mathrm{Cr}_{2} \mathrm{~S}_{4}$, die über ein dunkelbraunes Salz zu dem grünen Komplex $\left[\left(\mathrm{C}_{5} \mathrm{Me}_{5}\right)_{2} \mathrm{Cr}_{2} \mathrm{~S}_{4} \mathrm{Me}^{+} \mathrm{PF}_{6}{ }^{-}\right.$(IV) führt, dessen Methylresonanzen im ${ }^{1} \mathrm{H}-\mathrm{NMR}$-Spektrum (Tab. 2) bei $2.16\left(\mathrm{CH}_{3}\right)$ bzw. $1.51 \mathrm{ppm}\left(\mathrm{SCH}_{3}\right)$ liegen.

Bei der Methylierung von $\left(\mathrm{C}_{5} \mathrm{Me}_{5}\right)_{2} \mathrm{Cr}_{2} \mathrm{~S}_{5}$ bleibt die $\mathrm{S}_{5}$-Grundstruktur ebenso wie beim protonierten Komplex II bestehen, wie das FD-Massenspektrum von V bestätigt. Auch hier nimmt bei höherer Fadenheizung die Tendenz zur Schwefelabspaltung zu. Hieraus und aus der Elementaranalyse lässt sich auf eine Zusammensetzung $\left[\left(\mathrm{C}_{5} \mathrm{Me}_{5}\right)_{2} \mathrm{Cr}_{2} \mathrm{~S}_{5} \mathrm{Me}^{+} \mathrm{PF}_{6}^{-}\right.$schliessen. Das ${ }^{1} \mathrm{H}-\mathrm{NMR}-\mathrm{Spek}$ irum (Tab. 2) deutet ebenfalls auf eine Einfachmethylierung. Die vier auftretenden Signale lassen sich in zwei Paare bei 2.44 und 2.28 bzw. 1.65 und $2.22 \mathrm{ppm}$ separieren. deren Verhältnis zueinander durch Chromatographie an Sephadex LH 20 (Eluens Ethanol) oder Umkristallisation beeinflusst werden kann. Die Reinisolierung eines dieser Isomeren gelang jedoch bisher noch nicht. Diese Beobachtung lässt sich so interpretieren, dass nach der Primärmethylierung entweder des $\mu\left(\eta^{1}-S_{2}\right)$ - oder eines der beiden $\mu$-S-Liganden die Nukleophilie des gebildeten Kations so geschwächt wird. dass eine weitere Methylierung nicht mehr möglich ist.

Die Reaktion mit $\mathrm{C}_{5} \mathrm{H}_{5} \mathrm{Mn}(\mathrm{CO})_{2} \mathrm{THF}$

Die Umsetzung von $\left(\mathrm{C}_{3} \mathrm{Me}_{5}\right)_{2} \mathrm{Mo}_{2}\left(\mu-\mathrm{S}_{2}\right)(\mu-\mathrm{S})_{2}$ mit $\mathrm{C}_{5} \mathrm{H}_{5} \mathrm{Mn}(\mathrm{CO})_{2} \mathrm{THF}$ im Molverhältnis $1 / 1$ bei $15^{\circ} \mathrm{C}$ liefert das dunkelbraune Addukt $\left(\mathrm{C}_{5} \mathrm{Me}_{5}\right)_{2}\left(\mathrm{C}_{5} \mathrm{H}_{5}\right) \mathrm{Mo}_{2^{-}}$ $\mathrm{Mn}(\mathrm{CO})_{2} \mathrm{~S}_{4}$ (VI). Dagegen ergibt $\left(\mathrm{C}_{5} \mathrm{Me}_{5}\right)_{2} \mathrm{Cr}_{2} \mathrm{~S}_{5}$ unter den gleichen Bedingungen neben $\mathrm{C}_{5} \mathrm{H}_{5} \mathrm{Mn}(\mathrm{CO})_{3}$ ein Gemisch, das laut IR-, ${ }^{1} \mathrm{H}$-NMR- und Massenspektren den grünen Verbindungen $\left(\mathrm{C}_{5} \mathrm{H}_{5}\right)_{2} \mathrm{Mn}_{2}(\mathrm{CO})_{4} \mathrm{~S}_{2}$ und $\left(\mathrm{C}_{5} \mathrm{H}_{5}\right)_{2} \mathrm{Mn}_{2}(\mathrm{CO})_{4} \mathrm{~S}$ [7] zugeschrieben werden kann (Gl. 1). Zusammen mit unverbrauchtem Ausgangsmaterial wird noch $\left(\mathrm{C}_{5} \mathrm{Me}_{5}\right)_{2} \mathrm{Cr}_{2} \mathrm{~S}_{4}$ gefunden, was die Rolle von $\left(\mathrm{C}_{5} \mathrm{Me}_{5}\right)_{2} \mathrm{Cr}_{2} \mathrm{~S}_{5}$ als Schwefelüberträger erhärtet.

$$
\begin{aligned}
& \left(\mathrm{C}_{5} \mathrm{H}_{5}\right)_{2} \mathrm{Mn}_{2}(\mathrm{CO})_{4} \mathrm{~S}_{2}+\left(\mathrm{C}_{5} \mathrm{H}_{5}\right)_{2} \mathrm{Mn}_{2}(\mathrm{CO})_{4} \mathrm{~S}+\left(\mathrm{C}_{5} \mathrm{Me}_{5}\right)_{2} \mathrm{Cr}_{2} \mathrm{~S}_{4} \\
& \left(\mathrm{C}_{5} \mathrm{Me}_{5}\right)_{2} \mathrm{Cr}_{2} \mathrm{~S}_{5} \\
& \mathrm{C}_{5} \mathrm{H}_{5}(\mathrm{CO})_{2} \mathrm{MnTHF} \\
& \begin{aligned}
\left(\mathrm{C}_{5} \mathrm{Me}_{5}\right)_{2} \mathrm{Mo}_{2}\left(\mathrm{C}_{5} \mathrm{H}_{5}\right) \mathrm{Mn}(\mathrm{CO})_{2} \mathrm{~S}_{4} & \left(\mathrm{C}_{5} \mathrm{Me}_{5}\right)_{2} \mathrm{Mo}_{2} \mathrm{~S}_{4}
\end{aligned}
\end{aligned}
$$

Das IR-Spektrum (Tab. 1) von Komplex VI enthält neben den terminalen $\nu(C O)$-Frequenzen zahlreiche Absorptionen im Bereich unter $700 \mathrm{~cm}^{-1}$, der neben Metall-Carbonyl auch Metall-Schwefel-Schwingungen aufweist, so dass eine 
eindeutige Zuordnung nicht möglich ist. Das ${ }^{1} \mathrm{H}-\mathrm{NMR}$-Spektrum enthält je ein Singulett für die Methylprotonen (1.96 ppm) und die $\mathrm{C}_{5} \mathrm{H}_{5}$-Protonen (4.29 ppm). Die Integration über beide Signale ergibt ein Verhältnis von 30/5 Protonen und bestätigt somit die Zusammensetzung als 1/1-Addukt.

Bei der Diskussion der Struktur von VI ist zu berücksichtigen, dass zur Stabilisierung des ungesättigten $\mathrm{C}_{5} \mathrm{H}_{5} \mathrm{Mn}(\mathrm{CO})_{2}$-Fragments sowohl dessen Anlagerung an einen $\mu_{2}$-Schwefel [8] als auch an die $\mu_{2}-\mathrm{S}_{2}$-Gruppe unter symmetrischer Spaltung derselben erfolgen kann [9]. Beiden Vorschlägen A und B ist gemeinsam, dass die Gesamtelektronenbilanz der Ausgangsverbindung gewahrt bleibt und dass keine Mo-Mn-Bindungen benötigt werden. Um den Diamagnetismus von VI zu erklären, müsste wie in der Stammverbindung formal eine Mo-Mo-Doppelbindung angenommen werden. Während für VIA aufgrund der Tetraedergeometrie des Schwefels zwei Isomere denkbar sind (laut ${ }^{1} \mathrm{H}-\mathrm{NMR}$-Spektrum wird jedoch nur ein Isomer gebildet), scheint eine Anordnung, die wie in VIB von vier Sulfidbrücken ausgeht, auf den ersten Blick energetisch weniger günstig zu sein [10]. Die Umwandlung in $\mu_{3}$-Zentren könnte jedoch einen eventuell stabilisierenden Effekt besitzen. Eine endgültige Beantwortung dieser Frage ist noch nicht möglich, da VI bis jetzt keine Einkristalle liefert.

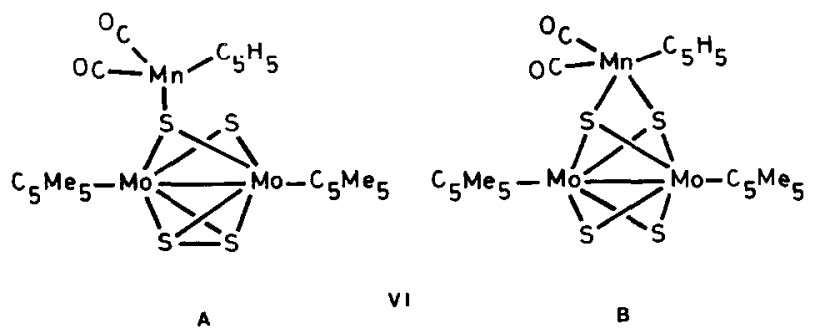

Hochdrucksynthesen mit Wasserstoff

Die Hydrierung der beiden Cr-Komplexe $\left(\mathrm{C}_{5} \mathrm{Me}_{5}\right)_{2} \mathrm{Cr}_{2} \mathrm{~S}_{5}$ bzw. $\left(\mathrm{C}_{5} \mathrm{Me}_{5}\right)_{2} \mathrm{Cr}_{2} \mathrm{~S}_{4}$ wurde im Autoklaven mit 150 bar $\mathrm{H}_{2}$ bei $80^{\circ} \mathrm{C}$ durchgeführt, wobei in beiden Fällen äusserst schwerlösliche, schwarzgrüne Kristalle isoliert werden konnten (Gl. 2). Sie besitzen laut Massenspektren und Elementaranalysen die Zusammensetzung $\left(\mathrm{C}_{5} \mathrm{Me}_{5}\right)_{4} \mathrm{Cr}_{4} \mathrm{~S}_{4}$. Dieser Komplex ist gemäss seiner 60 Valenzelektronenzahl in die Reihe der cubanartigen Cluster mit einem $\mathbf{M}_{4} \mathrm{~S}_{4}$-Kern einzureihen. Nach den

$$
\begin{aligned}
& \left(\mathrm{C}_{5} \mathrm{Me}_{5}\right)_{2} \mathrm{Cr}_{2} \mathrm{~S}_{5}, \stackrel{150 \mathrm{bar} \mathrm{H}_{2}}{\underset{80}{\circ} \mathrm{C}}\left(\mathrm{C}_{5} \mathrm{Me}_{5}\right)_{4} \mathrm{Cr}_{4} \mathrm{~S}_{4} \\
& \left(\mathrm{C}_{5} \mathrm{Me}_{5}\right)_{2} \mathrm{Cr}_{2} \mathrm{~S}_{4} \text { (I) }{ }^{80} \mathrm{C} \quad \text { (VII) } \\
& \left(\mathrm{C}_{5} \mathrm{Me}_{5}\right)_{2} \mathrm{Mo}_{2} \mathrm{~S}_{4} \underset{80^{\circ} \mathrm{C}}{\stackrel{150 \text { bar } \mathrm{H}_{2}}{\longrightarrow}}\left(\mathrm{C}_{5} \mathrm{Me}_{5}\right)_{2} \mathrm{Mo}_{2}(\mu-\mathrm{S})_{2}(\mu-\mathrm{SH})_{2}
\end{aligned}
$$

Überlegungen von Dahl et al. [11] sollte er einen Metalltetraeder mit $\mathrm{Cr}-\mathrm{Cr}$-Bindungen enthalten. Die Bestätigung dieses Vorschlags durch Röntgenstrukturanalyse schlug leider fehl [12]. Als Stützen für eine Strukturdiskussion können somit nur IRund Massenspektrum herangezogen werden. Sämtliche intensiven IR-Absorptionen der Ausgangsverbindung im Bereich zwischen 600 und $400 \mathrm{~cm}^{-1}$ sind zugunsten wesentlich schwächerer Absorptionen unter $400 \mathrm{~cm}^{-1}$ verschwunden. Dies kann am besten als eine Umfunktionierung der verschiedenen $\mu_{2}$-Schwefelliganden in $\mu_{3^{-}}$ 
Schwefelbrücken gedeutet werden unter gleichzeitigem Verlust von elementarem Schwefel bzw. Schwefelwasserstoff. Letzterer ist an seinem intensiven Geruch beim Öffnen des Autoklaven feststellbar. Für einen stabilen $\mathrm{M}_{4} \mathrm{~S}_{4}$-Kern spricht auch das Massenspektrum, in dem neben dem Molekülion und dessen doppelt geladenem Vertreter die sukzessive Abspaltung der $\mathrm{C}_{5} \mathrm{Me}_{5}$-Liganden dominiert. Ähnliches Fragmentierungsverhalten wurde auch an $\left(\mathrm{MeC}_{5} \mathrm{H}_{4}\right)_{4} \mathrm{Cr}_{4} \mathrm{~S}_{4}$ beobachtet, dessen Struktur kürzlich gesichert werden konnte [13].

Die Hochdruckhydrierung von $\left(\mathrm{C}_{5} \mathrm{Me}_{5}\right)_{2} \mathrm{Mo}_{2} \mathrm{~S}_{4}$ unter den gleichen Bedingungen wie oben liefert dagegen einen tiefroten Komplex der Zusammensetzung $\left(\mathrm{C}_{5} \mathrm{Me}_{5}\right)_{2} \mathrm{Mo}_{2} \mathrm{~S}_{4} \mathrm{H}_{2}$ (VIII). Dieser stimmt in seinen spektroskopischen Eigenschaften (Tab. 1, 2) mit einem aus $\left(\mathrm{C}_{5} \mathrm{Me}_{5}\right)_{2} \mathrm{Mo}_{2}(\mu \text {-S })_{2} \mathrm{~S}_{2}$ und Wasserstoff unter Normaldruck gewonnenen Komplex überein, der je zwei $\mu$-S und $\mu$-SH-Liganden enthält [6]. Obwohl $\left(\mathrm{C}_{5} \mathrm{Me}_{5}\right)_{2} \mathrm{Cr}_{2} \mathrm{~S}_{4}$ nicht wie der entsprechende Mo-Komplex unter $\mathrm{H}_{2^{-}}$ Aufnahme und SH-Brückenbildung reagiert, erscheint die Clusterbildung doch an die Gegenwart von $\mathrm{H}_{2}$ gebunden, wie ein weiteres Experiment zeigt: Erwärmt man eine Toluollösung von $\left(\mathrm{C}_{5} \mathrm{Me}_{5}\right)_{2} \mathrm{Cr}_{2} \mathrm{~S}_{5}$ auf $80^{\circ} \mathrm{C}$, so lässt sich selbst nach $17 \mathrm{~h}$ Reaktionsdauer nur der Ausgangskomplex zuruckgewinnen. Dagegen liefert die Druckreaktion mit 250 bar $\mathrm{H}_{2}$ bei Raumtemperatur einen dunkelvioletten, äusserst empfindlichen Komplex, dessen Lösungen stark nach $\mathrm{H}_{2} \mathrm{~S}$ riechen, dessen IRSpektrum in Substanz jedoch keine $\nu(\mathrm{SH})$-Schwingungen aufweist. Bei Versuchen zur Reinigung erhält man die Ausgangsverbindung verunreinigt durch $\left(\mathrm{C}_{5} \mathrm{Me}_{5}\right)_{2} \mathrm{Cr}_{2} \mathrm{~S}_{4}$ zurück.

\section{Diskussion}

Aus der Reaktivität der Komplexe $\left(\mathrm{C}_{5} \mathrm{Me}_{5}\right)_{2} \mathrm{Mo}_{2} \mathrm{~S}_{4}, \quad\left(\mathrm{C}_{5} \mathrm{Me}_{5}\right)_{2} \mathrm{Cr}_{2} \mathrm{~S}_{4}$ und $\left(\mathrm{C}_{5} \mathrm{Me}_{5}\right)_{2} \mathrm{Cr}_{2} \mathrm{~S}_{5}$ gegenüber den Elektrophilen $\mathrm{CF}_{3} \mathrm{CO}_{2} \mathrm{H}$ und $\mathrm{CH}_{3} \mathrm{I}$ lässt sich eine abgestufte Nukleophilie der Brückenschwefelliganden ableiten. $\left(\mathrm{C}_{5} \mathrm{Me}_{5}\right)_{2} \mathrm{Cr}_{2} \mathrm{~S}_{5}$ ist als einziger Komplex protonierbar, $\mathrm{d}$. h. als Reaktionspartner bietet sich nur die $\mu\left(\eta^{1}-\mathrm{S}_{2}\right)$-Gruppe an. Die Reaktion mit $\mathrm{CH}_{3} \mathrm{I}$ lässt sich als Methylierung der $\mu$-Sbzw. $\mu\left(\eta^{1}-S_{2}\right)$-Liganden interpretieren. Je ein Methylierungsprodukt ist ausgehend von $\left(\mathrm{C}_{5} \mathrm{Me}_{5}\right)_{2} \mathrm{Cr}_{2} \mathrm{~S}_{4}$ und $\left(\mathrm{C}_{5} \mathrm{Me}_{5}\right)_{2} \mathrm{Mo}_{2} \mathrm{~S}_{4}$ isolierbar. Die $\eta^{2}-\mathrm{S}_{2}-$ Brücke bleibt dagegen intakt, sie ist wohl nur über eine reduktive Spaltung der S-S-Bindung mit nachfolgender $\mathrm{CH}_{3} \mathrm{I}$-Reaktion anzugreifen [14].

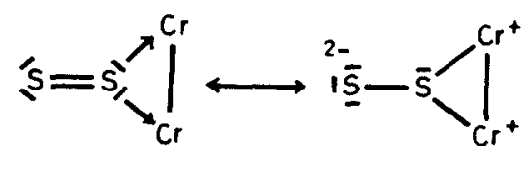

A

$\mathbf{B}$

Je nachdem, ob man den iso- $\mu\left(\eta^{1}-S_{2}\right)$-Liganden als 4- oder 2-Elektronendonor auffasst, erhält man die beiden Grenzstrukturen A und B. Die entsprechenden Bindungsparameter der Röntgenstrukturanalyse [1] deuten auf einen Zustand näher an Formulierung B: Beide $\mathrm{Cr}-\mathrm{S}-\mathrm{S}$-Winkel liegen mit jeweils $108.8^{\circ}$ im Bereich tetraedrischer Koordination, die S-S-Bindungslänge (2.101 $\AA$ ) ist signifikant länger als in anderen $\mu, \eta^{1}-S_{1}$-Liganden [15]. Dieser Befund entspräche einer Rückbindung aus besetzten Metall- $d$-Orbitalen in unbesetzte $p \pi^{\star}$-Zustände des $\mathrm{S}_{2}{ }^{2-}$-Liganden. Aus diesem Grund sollte das bisher nicht koordinierte Ende auch ein hervorragender 
Komplexligand sein. Der direkte Nachweis für eine Stabilisierung des kompletten Liganden am koordinativ ungesättigten $\mathrm{C}_{5} \mathrm{H}_{5} \mathrm{Mn}(\mathrm{CO})_{2}$-Fragment gelingt jedoch nicht. Allerdings lässt sich das Auftreten schwefelhaltiger Mn-Komplexe zusammen mit $\left(\mathrm{C}_{5} \mathrm{Me}_{5}\right)_{2} \mathrm{Cr}_{2} \mathrm{~S}_{4}$ als Hinweis auf die Auffüllung der am Mn-Atom vorhandenen Koordinationslücke werten. Ein ursprünglich eventuell gebildeter $\mu_{3}\left(\eta^{1}-S_{2}\right)$-Ligand könnte demnach unter Zurücklassung von $\left(\mathrm{C}_{5} \mathrm{Me}_{5}\right)_{2} \mathrm{Cr}_{2} \mathrm{~S}_{4}$ und eines reaktiven $\mathrm{C}_{5} \mathrm{H}_{5} \mathrm{Mn}(\mathrm{CO})_{2} \mathrm{~S}$-Bruchstücks auseinanderbrechen, das 7.u $\left[\mathrm{C}_{5} \mathrm{H}_{5}(\mathrm{CO})_{2} \mathrm{Mn}\right]_{2} \mathrm{~S}_{2}$ hzw. $\mathrm{C}_{5} \mathrm{H}_{5}(\mathrm{CO})_{2} \mathrm{Mn}_{2} \mathrm{~S}[10]$ rekombinieren kann.

Weniger klar ist der Ablauf der Hochdruckhydrierungen. Die Tatsache, dass die Clusterbildung von $\left(\mathrm{C}_{5} \mathrm{Me}_{5}\right)_{4} \mathrm{Cr}_{4} \mathrm{~S}_{4}$ von den beiden Chromkomplexen $\left(\mathrm{C}_{5} \mathrm{Me}_{5}\right)_{2} \mathrm{Cr}_{2} \mathrm{~S}_{4}$ und $\left(\mathrm{C}_{5} \mathrm{Me}_{5}\right)_{2} \mathrm{Cr}_{2} \mathrm{~S}_{5}$ ausgehend erfolgt, während $\left(\mathrm{C}_{5} \mathrm{Me}_{5}\right)_{2} \mathrm{Mo}_{2} \mathrm{~S}_{4}$ nur unter einfacher Wasserstoffaufnahme abreagiert, beweist lediglich, dass die Clusterbildung weniger von reaktiven Strukturelementen geprägt wird (die Ausbeuten sind für die beiden Cr-Komplexe nahezu gleich) als von der Natur des Zentralmetalls. Fest steht ausserdem, dass neben der Anwesenheit von Wasserstoff die thermische Zersetzung etwaiger Zwischenprodukte eine Rolle zu spielen scheint. Wie vorläufige Untersuchungen zeigen, lassen sich diese in Abfangreaktionen mit Kohlenmonoxid stabilisicren.

\section{Experimenteller Teil}

Alle Arbeiten wurden unter Luftausschluss und unter Verwendung von trockenen, $\mathrm{N}_{2}$-gesättigten Lösungsmitteln durchgeführt. Die Elementaranalysen (Tab. 3) wurden vom Mikroanalytischen Laboratorium der Universität Regensburg und von den Analytischen Laboratorien Malissa \& Reuter, D-5250 Engelskirchen (S) ausgeführt. $\left(\mathrm{C}_{5} \mathrm{Me}_{5}\right)_{2} \mathrm{Cr}_{2} \mathrm{~S}_{5}$ und $\left(\mathrm{C}_{5} \mathrm{Me}_{5}\right)_{2} \mathrm{Mo}_{2} \mathrm{~S}_{4}$ wurden aus $\left[\mathrm{C}_{5} \mathrm{Me}_{5}(\mathrm{CO})_{2} \mathrm{M}\right]_{2}(\mathrm{M}=\mathrm{Cr}, \mathrm{Mo})$ und elementarem Schwefel nach einem bereits beschriebenen Verfahren erhalten [1,2], ebenso $\mathrm{C}_{5} \mathrm{H}_{5} \mathrm{Mn}(\mathrm{CO})_{2}$ THF durch Bestrahlen einer THF-Lösung von $\mathrm{C}_{5} \mathrm{H}_{5} \mathrm{Mn}(\mathrm{CO})_{3}[7]$.

\section{Darstellung von $\left(\mathrm{C}_{5} \mathrm{Me}_{5}\right)_{2} \mathrm{Cr}_{2} \mathrm{~S}_{4}(\mathrm{I})$}

Eine Mischung aus $160 \mathrm{mg}(0.3 \mathrm{mmol})\left(\mathrm{C}_{5} \mathrm{Me}_{5}\right)_{2} \mathrm{Cr}_{2} \mathrm{~S}_{5}, 79 \mathrm{mg}(0.3 \mathrm{mmol})$ Triphenylphosphin und $100 \mathrm{ml}$ Toluol wird $20 \mathrm{~h}$ bei $45^{\circ} \mathrm{C}$ gerührt. Nach Konzentrieren auf $10 \mathrm{ml}$ wird an $\mathrm{SiO}_{2}$ chromatographiert. Mit Toluol/Ft ${ }_{2} \mathrm{O}$ 10/1 isoliert man als einzige Zone den blaugrünen Komplex I, der aus Toluol/Pentan $1 / 1$ umkristallisiert wird.

$70 \mathrm{eV}-$ Massenspektrum $(m / e$, rel. Intensitäten in $\%): M^{+}=502(43),[M-2 \mathrm{~S}]^{+}$ $=438(100$, metastabiler Übergang 382.16$), M^{2+}=251(10)$.

\section{Protonierung von $\left(\mathrm{C}_{5} \mathrm{Me}_{5}\right)_{2} \mathrm{Cr}_{2} \mathrm{~S}_{5}$ mit $\mathrm{CF}_{3} \mathrm{CO}_{2} \mathrm{H}$}

Die Lösung von $81 \mathrm{mg}(0.15 \mathrm{mmol})\left(\mathrm{C}_{5} \mathrm{Me}_{5}\right)_{2} \mathrm{Cr}_{2} \mathrm{~S}_{5}$ wird mit $0.2 \mathrm{ml} \mathrm{CF}_{3} \mathrm{CO}_{2} \mathrm{H}$ versetzt, wobei ein grünes Öl ausfällt. Nach dem Entfernen des Lösungsmittels wird das zurückbleibende Öl in $15 \mathrm{ml} 96 \%$ igem Ethanol bei $40^{\circ} \mathrm{C}$ gelöst und mit $15 \mathrm{ml}$ Wasser sowie $50 \mathrm{mg} \mathrm{NH}{ }_{4} \mathrm{PF}_{6}$ versetzt. Der sofort ausfallende graugrüne Niederschlag wird filtriert und gründlich mit Wasser und Ether gewaschen. Ein analysenreines Produkt erhält man durch Umkristallisieren aus einem Gemisch Aceton/Methylenchlorid/Ethanol 6/1/1. 


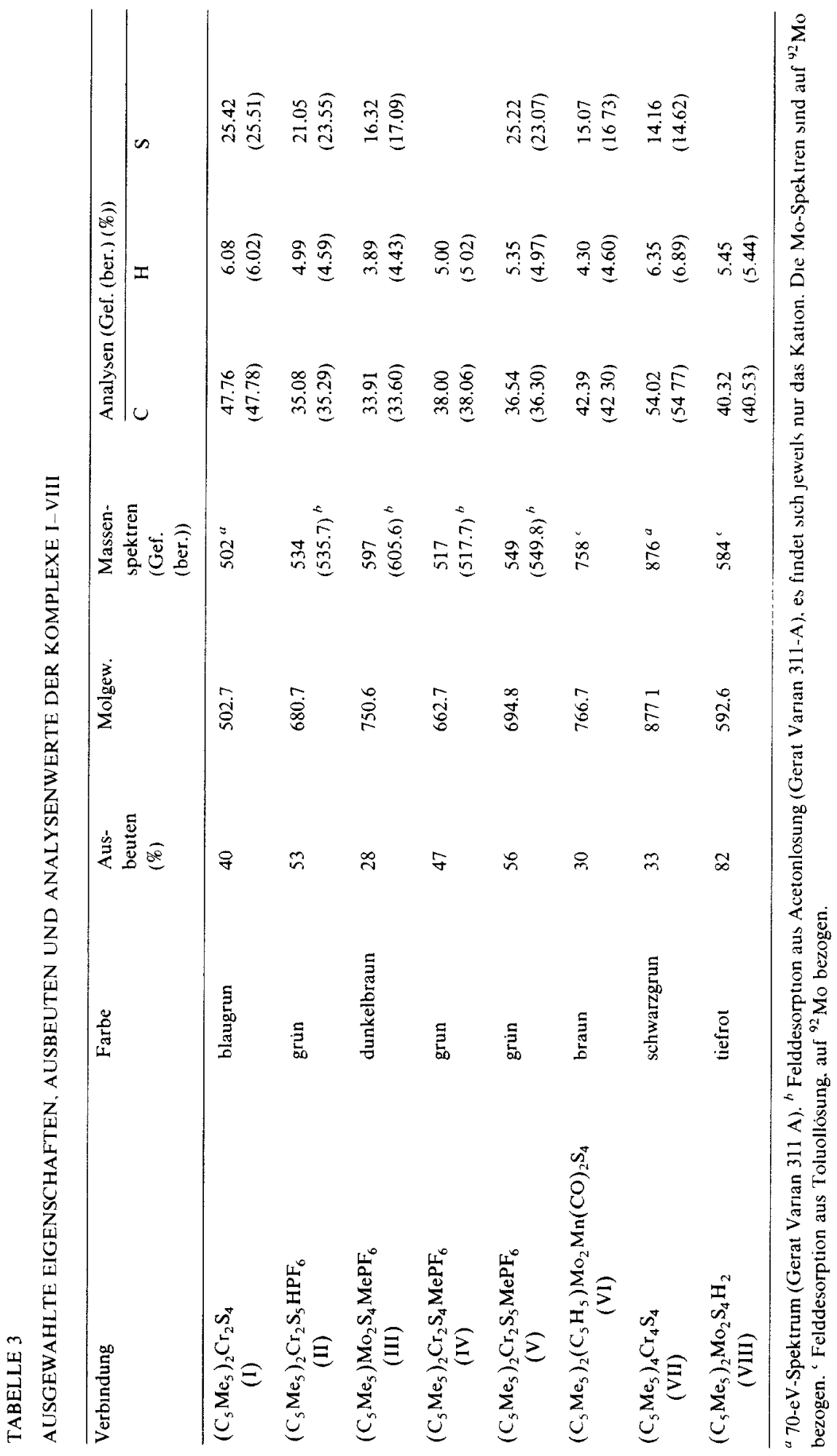


Allgemeine Arbeitsvorschrift zur Umsetzung der Komplexe $\left(\mathrm{C}_{5} \mathrm{Me}_{5}\right)_{2} \mathrm{Mo}_{2} \mathrm{~S}_{4}$, $\left(\mathrm{C}_{5} \mathrm{Me}_{5}\right)_{2} \mathrm{Cr}_{2} \mathrm{~S}_{4}$ und $\left(\mathrm{C}_{5} \mathrm{Me}_{5}\right)_{2} \mathrm{Cr}_{2} \mathrm{~S}_{5}$ mit $\mathrm{CH}_{3} \mathrm{I}$

$0.2 \mathrm{mmol}$ des betreffenden Komplexes werden in $30 \mathrm{ml}$ Toluol gelöst, mit $0.1 \mathrm{ml}$ Methyliodid versetzt und noch 15 min gerührt. Die auftretenden Niederschläge (dunkelgrün im Fall von Mo, dunkelbraun für die beiden $\mathrm{Cr}$-Komplexe) werden abfiltriert, in möglichst wenig 96\%igem Ethanol gelöst und mit der gleichen Menge Wasser versetzt. Die nach Zugabe von $100 \mathrm{mg} \mathrm{NH}_{4} \mathrm{PF}_{6}$ ausfallenden $\mathrm{PF}_{6}$-Salze III-V werden abfiltriert und gründlich mit Wasser und Ether gewaschen. Analysenreine Produkte erhält man durch Umkristallisieren aus Aceton/Ether 1/1.

Darstellung van $\left(\mathrm{C}_{5} \mathrm{Me}_{5}\right)_{2}\left(\mathrm{C}_{5} \mathrm{H}_{5}\right) \mathrm{Mo}_{2} \mathrm{Mn}(\mathrm{CO})_{2} \mathrm{~S}_{4}(\mathrm{VI})$

Die rote Lösung von $1.0 \mathrm{mmol}_{5} \mathrm{H}_{5} \mathrm{Mn}(\mathrm{CO})_{2}$ (THF) in $230 \mathrm{ml}$ Tetrahydrofuran (THF) wird langsam und unter Wasserkühlung zur dunkelblauen Lösung von 245 $\mathrm{mg}(0.5 \mathrm{mmol})\left(\mathrm{C}_{5} \mathrm{Me}_{5}\right)_{2} \mathrm{Mo}_{2}\left(\mu-\mathrm{S}_{2}\right)(\mu-\mathrm{S})_{2}$ in $50 \mathrm{ml}$ THF getropft. Es wird noch ca. $16 \mathrm{~h}$ gerührt, wobei sich eine allmähliche Farbänderung nach dunkelbraun zeigt. Nach dem Entfernen des Lösungsmittels wird der dunkelbraune Rückstand in 100 $\mathrm{ml}$ Toluol aufgenommen und an $\mathrm{SiO}_{2}($ Säule $40 \times 3.5 \mathrm{~cm}$ ) chromatographiert. Mit Toluol/Petrolether $\left(40-60^{\circ} \mathrm{C}\right) 1 / 1$ eluiert man der Reihe nach $\mathrm{C}_{5} \mathrm{H}_{5} \mathrm{Mn}(\mathrm{CO})_{3}$ als gelbe Zone, eine schwach grüne bzw. schwach rote Zone, beide aufgrund zu geringer Substanzmengen nicht charakterisierbar, und das gewünschte Produkt als dunkelbraune Zone. Den analysenreinen Komplex VI erhält man durch Umkristallisation aus Toluol/Ether/Pentan $10 / 3 / 4$ bei $-18^{\circ} \mathrm{C}$.

\section{Umsetzung von $\left(\mathrm{C}_{5} \mathrm{Me}_{5}\right)_{2} \mathrm{Cr}_{2} \mathrm{~S}_{5}$ mit $\mathrm{C}_{5} \mathrm{H}_{5} \mathrm{Mn}(\mathrm{CO})_{2} \mathrm{THF}$}

$108 \mathrm{mg}(0.2 \mathrm{mmol})\left(\mathrm{C}_{5} \mathrm{Me}_{5}\right)_{2} \mathrm{Cr}_{2} \mathrm{~S}_{5}$ werden wie oben mit der äquimolaren Menge des $\mathrm{C}_{5} \mathrm{H}_{5} \mathrm{Mn}(\mathrm{CO})_{2}$ THF-Komplexes umgesetzt und noch etwa $16 \mathrm{~h}$ gerührt. Dabei tritt eine allmähliche Farbänderung von hellbraun nach dunkelgrün ein. Bei der Chromatographie an $\mathrm{SiO}_{2}$ eluiert man zunächst mit Toluol gelbes $\mathrm{C}_{5} \mathrm{H}_{5} \mathrm{Mn}(\mathrm{CO})_{3}$ und anschliessend eine grüne Zone, die das Produktgemisch $\left[\mathrm{C}_{5} \mathrm{H}_{5}(\mathrm{CO})_{2} \mathrm{Mn}\right]_{2^{-}}$ $\mathrm{S}_{2} /\left[\mathrm{C}_{5} \mathrm{H}_{5}(\mathrm{CO})_{2} \mathrm{Mn}\right]_{2} \mathrm{~S}$ im Verhältnis $1 / 1$ enthält $\left(35 \mathrm{mg}\right.$, Nachweis IR- und ${ }^{1} \mathrm{H}$ NMR-spektroskopisch [10] sowie FD-Massenspektrum aus Toluollösung mit Molekülionen bei 416 und 384). Eine nachfolgende, mit Toluol/Ether $25 / 1$ eluierte Zone enthält laut ${ }^{1} \mathrm{H}-\mathrm{NMR}$ - und IR-Spektrum ein Gemisch aus $\left(\mathrm{C}_{5} \mathrm{Me}_{5}\right)_{2} \mathrm{Cr}_{2} \mathrm{~S}_{5}$ und $\left(\mathrm{C}_{5} \mathrm{Me}_{5}\right)_{2} \mathrm{Cr}_{2} \mathrm{~S}_{4}$ im ungefähren Verhältnis 2/3.

\section{Darstellung von $\left(\mathrm{C}_{5} \mathrm{Me}_{5}\right)_{4} \mathrm{Cr}_{4} \mathrm{~S}_{4}$ (VII)}

Die Lösung von $315 \mathrm{mg}(0.6 \mathrm{mmol})\left(\mathrm{C}_{5} \mathrm{Me}_{5}\right)_{2} \mathrm{Cr}_{2} \mathrm{~S}_{5}$ in $20 \mathrm{ml}$ Toluol wird in einem 100-ml-Rollautoklaven nach mehrmaligem Spülen mit Wasserstoff mit 150 bar $\mathrm{H}_{2}$ bei $80^{\circ} \mathrm{C}$ zur Reaktion ( $24 \mathrm{~h}$ ) gebracht. Nach Abkühlen auf Raumtemperatur bilden sich schwarzgrüne Kristalle von VII, die abfiltriert und mit Pentan gewaschen werden.

70-eV-Massenspektrum $\left(m / e\right.$,(rel. Intensitäten in \%)): $M^{+}=876(100),[M-$ $\left.\mathrm{CH}_{4}\right]^{+}=860(17),\left[M-\mathrm{C}_{5} \mathrm{Me}_{5}\right]^{+}=741(21),\left[M-\mathrm{C}_{5} \mathrm{Me}_{5}-\mathrm{CH}_{4}\right]^{+}=752(2),[M-$ $\left.2 \mathrm{C}_{5} \mathrm{Me}_{5}\right]^{+}=606(21),\left[M-2 \mathrm{C}_{5} \mathrm{Me}_{5}-\mathrm{CH}_{4}\right]^{+}=590(4),\left[M-3 \mathrm{C}_{5} \mathrm{Me}_{5}\right]^{+}=471$ (32), $\left[M-3 \mathrm{C}_{5} \mathrm{Me}_{5}-\mathrm{CH}_{4}\right]^{+}=455(2), M^{2+}=438(42),\left[M-4 \mathrm{C}_{5} \mathrm{Me}_{5}\right]-336(11)$.

Darstellung von $\left(\mathrm{C}_{5} \mathrm{Me}_{5}\right)_{2} \mathrm{Mo}_{2} \mathrm{~S}_{4} \mathrm{H}_{2}$ (VIII)

Die dunkelblaue Lösung von $354 \mathrm{mg}(0.6 \mathrm{mmol})\left(\mathrm{C}_{5} \mathrm{Me}_{5}\right)_{2} \mathrm{Mo}_{2} \mathrm{~S}_{4}$ in $20 \mathrm{ml}$ Toluol 
wird in einem $100 \mathrm{ml}$ Rollautoklaven nach mehrmaligem Spülen mit $\mathrm{H}_{2}$ mit 150 bar $\mathrm{H}_{2}$ bei $80^{\circ} \mathrm{C}$ zur Reaktion ( $24 \mathrm{~h}$ ) gebracht. Die nunmehr tiefrote Lösung wird nach dem Abkühlen an $\mathrm{SiO}_{2}$ (Säule $20 \times 3 \mathrm{~cm}$ ) chromatographiert. Das Produkt wird mit Toluol als dunkelrote Bande eluiert. Umkristallisieren aus Toluol bei $-35^{\circ} \mathrm{C}$ ergibt analysenreine Kristalle von VIII.

\section{Dank}

Wir danken Herrn M. Muschiol für die Durchführung der Hochdruckversuche.

\section{Literatur}

1 H. Brunner, J. Wachter, E. Guggolz und M.L. Ziegler. J. Am. Chem. Soc., 104 (1982) 1765.

2 H. Brunner, W. Meier, J. Wachter, E. Guggolz, T. Zahn und M.L. Ziegler, Organometallıcs, 1 (1982) 1107.

3 H. Brunner und J. Wachter, J. Organomet. Chem., 240 (1982) C41: H. Brunner. H. Kauermann und J. Wachter, Angew. Chem., 95 (1983) 567; Angew. Chem., Int. Ed. Engl., 22 (1983) 549.

4 C.M. Bolinger, T.B. Rauchfuss und A L. Rheingold, Organometallics, 1 (1982) 1551.

5 C.M. Bolinger, T.B. Rauchfuss und A.L. Rheingold, J. Am. Chem. Soc., im Druck.

6 M. Rakowskı DuBois, M.C. VanDerveer, D.L. DuBors, R.C. Haltiwanger und W.K. Miller, J. Am. Chem. Soc., 102 (1980) 7456.

7 M. Herberhold, D. Reiner, B. Zimmer-Gasser und U. Schubert, Z. Naturforsch. B, 35 (1980) 1281.

8 A.A. Pasynskı1, I.L. Eremenko, B. Orazsakhatov, V.T. Kalınnikov, G.G. Aleksandrov und Y.T. Struchkov, J. Organomet. Chem.. 216 (1981) 211.

9 S.A. Khattab, L. Markó, G. Bor und B. Markó, J. Organomet. Chem., 1 (1964) 373; D. Seyferth, R.S. Henderson und M.K. Gallagher, J. Organomet. Chem., 193 (1980) C75: V.W. Day, D.A. Lesch und T.B. Rauchfuss, J. Am. Chem. Soc., 104 (1982) 1290.

10 D.L. DuBois, W.K. Miller und M. Rakowski DuBois, J. Am. Chem. Soc., 103 (1981) 3429.

11 'I. Toan, R. Broach und L.F. Dahl, VI. Int. Conf. Organomet. Chem., Amherst 1973.

12 Prof. M.L. Ziegler sind wir fur Diffraktometermessungen an Einkristallen von VII zu Dank verpflichtet.

13 A.A. Pasynskii, I.L. Eremenko, Y.V. Rakıtın, V.M. Novotortsev, O G. Ellert. V.T. Kalinnıkov, V.E. Shklover, Y.T. Struchkov, S.V. Lindeman, T.K. Kurbanov und G.S. Gasanov, J. Organomet. Chem., 248 (1983) 309.

14 D. Seyferth, R.S. Henderson und I.-C. Song, I. Organomet. Chem., 192 (1980) C1.

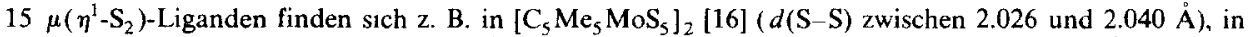
$\left(\mathrm{MeC}_{5} \mathrm{H}_{4}\right)_{2} \mathrm{~V}_{2} \mathrm{~S}_{5}[4](d(\mathrm{~S}-\mathrm{S}) 2.023 \AA)$ und in $\left[\mathrm{C}_{5} \mathrm{H}_{5} \mathrm{Mn}(\mathrm{CO})_{2}\right]_{2} \mathrm{~S}_{2}[7](d(\mathrm{~S}-\mathrm{S}) 2.007 \AA)$. Weitere Bespiele in A. Múller und W. Jaegermann, Inorg. Chem., 18 (1979) 2631.

16 M. Rakowskı DuBors, D L DuBois. M.C. VanDerveer und R.C. Haltiwanger, Inorg. Chem , 20 (1981) 3064. 